\title{
Why does pulmonary vein thrombus predominantly develop after left upper lobectomy?
}

\author{
Tadashi Umehara ${ }^{1}$, Koji Takumi ${ }^{2}$, Kazuhiro Ueda ${ }^{1}$, Masami Sato ${ }^{1}$ \\ ${ }^{1}$ Department of General Thoracic Surgery, Kagoshima University Graduate School of Medical and Dental Sciences, 8-35-1 Sakuragaoka, Kagoshima \\ 890-8520, Japan; ${ }^{2}$ Department of Radiology, Kagoshima University Graduate School of Medical and Dental Sciences, 8-35-1 Sakuragaoka, \\ Kagoshima 890-8520, Japan \\ Correspondence to: Kazuhiro Ueda, MD, PhD. Department of General Thoracic Surgery, Kagoshima University Graduate School of Medical and \\ Dental Sciences, 8-35-1 Sakuragaoka, Kagoshima 890-8520, Japan. Email: k7433286@kadai.jp. \\ Response to: Sekine T, Nakaza M, Kumita S. Careful consideration should be paid in the new imaging modality evaluation. J Thorac Dis 2021;13:422-4.
}

Submitted Nov 27, 2020. Accepted for publication Dec 24, 2020.

doi: $10.21037 /$ jtd-20-3411

View this article at: http://dx.doi.org/10.21037/jtd-20-3411

It is my great honor to express my opinion on the editorial commentary (1) regarding our paper, 'Four-dimensional flow magnetic resonance imaging study to explain high prevalence of pulmonary vein stump thrombus after left upper lobectomy' (2). Firstly, we observed dynamic blood movement in the left atrium after lung lobectomy with four-dimensional flow magnetic resonance imaging (4D MRI) and identified some characteristic hemodynamic features that are predominantly found after left upper lobectomy (LUL). Most importantly, LUL likely resulted in extensive to-and-fro blood movement near the pulmonary vein (PV) stump. Because PV thrombus develops almost exclusively after LUL, our results may warrant a further study on the real risk of pulmonary vein thrombus.

As was pointed out by Dr. Sekine et al., the heart phase of our protocol may be relatively small for $4 \mathrm{D}$ flow analyses and may underestimate some 4D flow parameters. We should have mentioned this point in the discussion section as a limitation of our study. However, we do not think that our protocol had a significant impact on our results. The flow pattern and flow rate of the cases in which LUL was performed were obviously different from the cases in which other lobectomy was performed, as shown in the results. We believe that the difference in the standard deviation (SD) of the blood flow velocity near the PV stump between patients who underwent LUL and those who underwent other lobectomy may be further emphasized by an evaluation with higher temporal resolution. According to Figure 2A,B,C,D, some patients certainly had a heart phase of 8 or 9 because of the variation in the heart rate. However, please note that Figure 2A shows the results of 13 patients who underwent LUL and Figure 2B,C,D show the results of different patients who underwent different operations.

With regard to the blood flow velocity near the venous stump, we agree that the values may differ greatly according to the position of the ROIs. In our study, we strictly placed the ROIs at the junction between the pulmonary vein and left atrium to minimize the measurement error. As shown in Figure 4, we found a significant positive correlation between the SD of the velocity and the length of the PV stump in our overall patients. Although multiple factors may influence the SD of the velocity, our data may partly suggest that the SD of the velocity becomes lower when the ROI is placed near the PV stump, which is in accordance with Dr. Sekine's suggestion (Figure 1).

We proposed five characteristic flow patterns in the left atrium in the Results section (Table 2). It appeared that three of the five flow patterns promoted to-and-fro blood movement near the PV stump (green color on Figure 1B,C,D), which seemed to generate blood turbulence, while the remaining two flow patterns promoted washing out of the PV stump. These subjective findings are described in the Discussion section. We are well aware that we must validate the findings with additional cases to clarify whether they are indeed associated with PV thrombus or not. Although we did not include patients with 
paroxysmal atrial fibrillation in our study, a previous study suggested that a large vortex in the left atrium in patients with paroxysmal atrial fibrillation was associated with a low risk of thrombus (3). Whether or not large vortex in the left atrium, as was observed in our patients, reduces the risk of PV stump thrombus should be clarified in a future study.

We also agree that the SD of the velocity is not correlated with the grade of turbulence. However, when the velocity was evaluated near the PV stump, the direction of blood flow (antegrade or retrograde) changes extensively, generating to-and-fro movement, particularly after LUL. This finding was reproduced by Dr. Sekine et al., as can be seen in Figure 1. We believe that the SD is a useful parameter for quantifying the grade of to-and-fro blood movement. Nonetheless, it remains controversial if a high SD of the velocity is associated with a high risk of PV stump thrombus because we did not compare the SD between patients who developed PV stump thrombus and those who did not. Interestingly, although we experienced two patients with PV stump thrombus after LUL, these two patients did not show the highest SD values among patients who had undergone LUL. The impact of the SD on PV stump thrombus will be clarified in our next study.

Before publishing our report, Matsumoto et al. published the first report on a patient who experienced PV stump thrombus after LUL by evaluating hemodynamics with 4D flow MRI (4). They suggested a reduction in the blood flow rate around the PV stump. However, concern remains if this finding is simply a result of lobectomy or not, because of the nature of a case study. We believe that our paper is the first to compare the 4D flow MRI findings of a high-risk population undergoing LUL with a low-risk population undergoing other lobectomy.

\section{Acknowledgments}

Funding: None.

\section{Footnote}

Provenance and Peer Review: This article was commissioned by the editorial office, Fournal of Thoracic Disease. The article did not undergo external peer review.

Conflicts of Interest: All authors have completed the ICMJE uniform disclosure form (available at http://dx.doi. org/10.21037/jtd-20-3441). The authors have no conflicts of interest to declare.

Ethical Statement: The authors are accountable for all aspects of the work in ensuring that questions related to the accuracy or integrity of any part of the work are appropriately investigated and resolved.

Open Access Statement: This is an Open Access article distributed in accordance with the Creative Commons Attribution-NonCommercial-NoDerivs 4.0 International License (CC BY-NC-ND 4.0), which permits the noncommercial replication and distribution of the article with the strict proviso that no changes or edits are made and the original work is properly cited (including links to both the formal publication through the relevant DOI and the license). See: https://creativecommons.org/licenses/by-nc-nd/4.0/.

\section{References}

1. Sekine T, Nakaza M, Kumita S. Careful consideration should be paid in the new imaging modality evaluation. J Thorac Dis 2021;13:422-4. doi: 10.21037/jtd-20-3229

2. Umehara T, Takumi K, Ueda K, et al. Four-dimensional flow magnetic resonance imaging study to explain high prevalence of pulmonary vein stump thrombus after left upper lobectomy. J Thorac Dis 2020;12:5542-51.

3. Garcia J, Sheitt H, Bristow MS, et al. Left atrial vortex size and velocity distributions by $4 \mathrm{D}$ flow MRI in patients with paroxysmal atrial fibrillation: Associations with age and CHA2 DS2 -VASc risk score. J Magn Reson Imaging 2020;51:871-84.

4. Matsumoto M, Takegahara K, Inoue T, et al. 4D Flow MR Imaging Reveals a Decrease of Left Atrial Blood Flow in a Patient with Cardioembolic Cerebral Infarction after Pulmonary Left Upper Lobectomy. Magn Reson Med Sci 2020;19:290-3.
Cite this article as: Umehara T, Takumi K, Ueda K, Sato M. Why does pulmonary vein thrombus predominantly develop after left upper lobectomy? J Thorac Dis 2021;13(1):420-421. doi: 10.21037/jtd-20-3411 